\title{
Catecholaminergic polymorphic ventricular tachycardia: electrocardiographic characteristics and optimal therapeutic strategies to prevent sudden death
}

\author{
N Sumitomo, K Harada, M Nagashima, T Yasuda, Y Nakamura, Y Aragaki, A Saito, \\ K Kurosaki, K Jouo, M Koujiro, S Konishi, S Matsuoka, T Oono, S Hayakawa, M Miura, \\ H Ushinohama, T Shibata, I Niimura
}

See end of article for authors' affiliations

Correspondence to: Dr Naokata Sumitomo Department of Paediatrics Nihon University School of Medicine, 30-1 Oyaguchi Kamimachi, Itabashi, Tokyo 173-8610, Japan;

¡†9n-smtm@asahi-net.or.jp

Accepted 28 August 2002
Objective: To investigate the clinical outcome, ECG characteristics, and optimal treatment of catecholaminergic polymorphic ventricular tachycardia (CPVT), a malignant and rare ventricular tachycardia. Patients and methods: Questionnaire responses and ECGs of 29 patients with CPVT were evaluated. Mean (SD) age of onset was $10.3(6.1)$ years.

Results: The initial CPVT manifestations were syncope $(79 \%)$, cardiac arrest $(7 \%)$, and a family history (14\%). ECGs showed sinus bradycardia and a normal QTc. Mean heart rate during CPVT was 192 (30) beats $/ \mathrm{min}$. Most cases were non-sustained $(72 \%)$, but $21 \%$ were sustained and $7 \%$ were associated with ventricular fibrillation. The morphology of CPVT was polymorphic (62\%), polymorphic and bidirectional $(21 \%)$, bidirectional $(10 \%)$, or polymorphic with ventricular fibrillation $(7 \%)$. There was $100 \%$ inducement of CPVT by exercise, $75 \%$ by catecholamine infusion, and none by programmed stimulation. No late potential was recorded. Onset was in the right ventricular outflow tract in more than half the cases. During a follow up of 6.8 (4.9) years, sudden death occurred in $24 \%$ of the patients, $7 \%$ of whom had anoxic brain damage. Autosomal dominant inheritance was seen in $8 \%$ of the patients' families. $\beta$ Blockers completely controlled CPVT in only $31 \%$ of cases. Calcium antagonists partially suppressed CPVT in autosomal dominant cases.

Conclusions: CPVT may arise in certain distinct areas but the prognosis is poor. The onset of CPVT may be an indication for an implanted cardioverter-defibrillator.
S nce Berg ${ }^{1}$ first reported multifocal ventricular extrasystoles with Adams-Stokes syndrome, several exercise related cases of polymorphic ventricular tachycardia have been reported. ${ }^{1-12}$ Leenhardt and colleagues described the clinical course and prognosis of 21 cases of this particular ventricular tachycardia, calling it catecholaminergic polymorphic ventricular tachycardia (CPVT). ${ }^{10}$ In general, monomorphic ventricular tachycardias arising in patients without structural cardiac abnormalities have a good prognosis. ${ }^{13} 14$ However, the prognosis, clinical features, and ECG characteristics of CPVT remain unclear. Our aim in this study was to examine the ECG characteristics, clinical outcome, and optimal treatment of CPVT.

Several factors define CPVT. First, more than two types of ventricular tachycardia morphology can be induced by exercise or catecholamine infusion during more than three consecutive beats. Second, there is absence of electrolyte imbalance, drug treatment, or organic heart disease such as cardiomyopathy, ischaemic heart disease, or congenital heart disease that could be a cause of polymorphic ventricular tachycardia or ventricular fibrillation. Finally, there is absence of primary electrical disease such as the long QT syndrome or the Brugada syndrome. ${ }^{15}$

\section{METHODS}

Questionnaires were sent to major Japanese paediatric cardiology centres. We selected 29 of 34 cases ( 13 male, 16 female) from 14 institutes that fulfilled our criteria. The other five cases who did not meet our criteria had exercise induced monomorphic ventricular tachycardia.

Each of the cases had a complete surface ECG recorded in the control state as well as during or at the onset of CPVT, which was then scanned and filed in 300 dpi, 256 grey scaled
JPEG files. ECG measurements were made using a 200\% magnification of these files. A cardiologist examined each case, and a chest $x$ ray and echocardiogram were also done.

Using the ECG taken at the initiation of CPVT during the exercise test, the origin of the CPVT was estimated from the bundle branch block pattern and the QRS axis.

\section{Statistics}

All numerical data are presented as mean (SD). Fisher's exact test was used to compare discrete variables. Depending on the type of variable and the sample numbers, further statistical analysis was done using a Mann-Whitney non-parametric test or a $\chi^{2}$ test as appropriate. The probability of remaining free of sudden cardiac death was estimated by the KaplanMeier method. A probability value of $p<0.05$ was considered significant.

\section{RESULTS}

\section{Patient background}

Mean (SD) age of onset of CPVT in the 29 patients was 10.3 (6.1) years (range 2-30 years). Only two of the patients had organic heart disease: one had mild pulmonary stenosis ( $10 \mathrm{~mm} \mathrm{Hg}$ pressure gradient between the main pulmonary artery and the right ventricle); the other had a non-operated vascular ring (right aortic arch and left subclavian artery anomaly).

CPVT was identified by syncope in 23 cases $(79 \%)$, by cardiac arrest in two (7\%), and through the family history in four ( $14 \%)$. One of the four familial cases experienced syncope during the follow up period and another had syncopal attacks during break time in junior high school; however, the exact cause remains unknown. Two of the four familial cases did not experience syncope. 


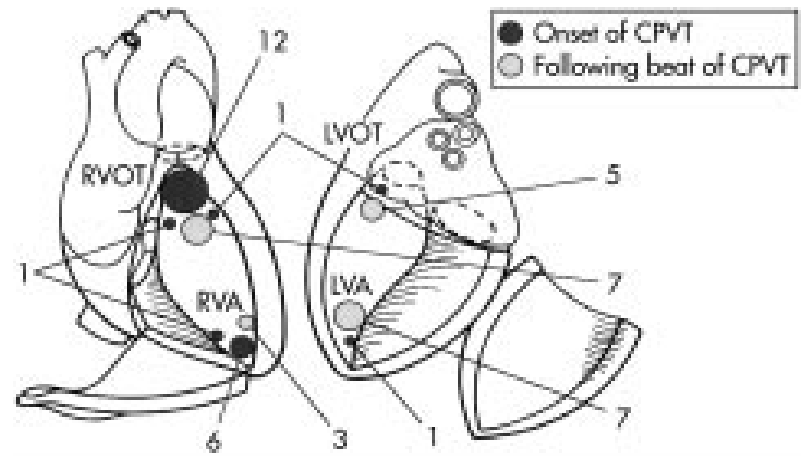

Figure 1 The onset of the foci and the following catecholaminergic polymorphic ventricular tachycardia (CPVT) beats in the study population. LVA, left ventricular apex; LVOT, left ventricular outflow tract; RVA, right ventricular apex; RVOT, right ventricular outflow tract.

\section{Resting ECG}

The ECG showed a tendency to sinus bradycardia (59 (11) beats/min), a normal QRS axis $\left(48(31)^{\circ}\right)$, a normal PR interval $(0.13(0.02) \mathrm{s})$, and a normal QTc $(0.40$ (0.02) s). There were no ST-T or T wave abnormalities in these cases.

The arrhythmias detected at rest were accelerated junctional rhythm in two cases, junctional escape in one case, and atrial tachycardia in one case.

\section{CPVT characteristics}

At the time of CPVT onset, QRS duration was relatively narrow, at $0.11(0.02) \mathrm{s}$ (range $0.08-0.13 \mathrm{~s})$, and the coupling interval was $0.33(0.05)$ s. The QT and QTc intervals immediately before ventricular tachycardia onset were $0.26(0.04) \mathrm{s}$ $(0.22-0.40 \mathrm{~s})$ and $0.33(0.05) \mathrm{s}(0.26-0.44 \mathrm{~s})$, respectively. The preceding sinus rate at CPVT onset was 161 (28) beats/min (79-214 beats/min).

CPVT onset was suggested by the QRS morphology of the first CPVT beat in 27 cases. The onset focus originated in the right ventricular outflow tract in 15 cases; in the right ventricular outflow tract and the left ventricular outflow tract in one case; in the right ventricular apex in six cases; in the right ventricular apex and the right ventricular outflow tract in three cases; in the left ventricular apex in one case; and in the left ventricular apex and the right ventricular outflow tract in one case (fig 1). Of the CPVT patients, most had a single unifocal site of origin ( 22 of 27 cases, $81 \%$ ), while 19\% (five of 27 cases) had double foci. The succeeding CPVT origin was left

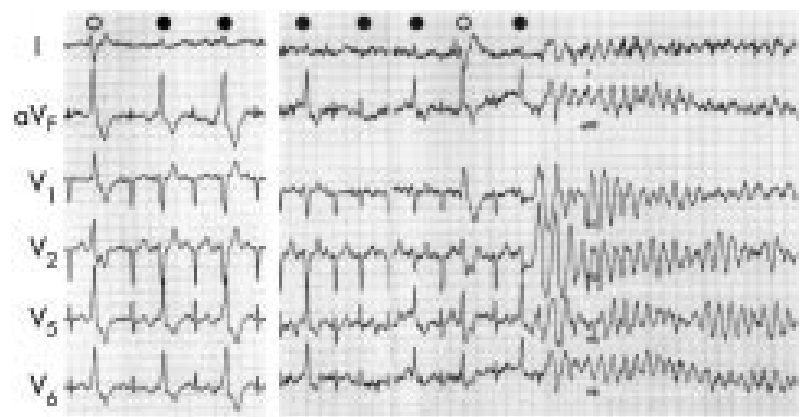

Figure 2 Exercise induced ventricular fibrillation. This is an ECG of a 13 year old girl who had syncope while running. During a treadmill exercise test, bigemini involving right bundle branch block and rightward axis (empty circle) and left bundle branch block, inferior axis type, premature ventricular contraction (filled circles) were induced (left panel). Ventricular fibrillation was induced after left bundle branch block type premature ventricular contraction. Several of the premature beat morphologies appear somewhat different because of the fusion of the sinus beat.
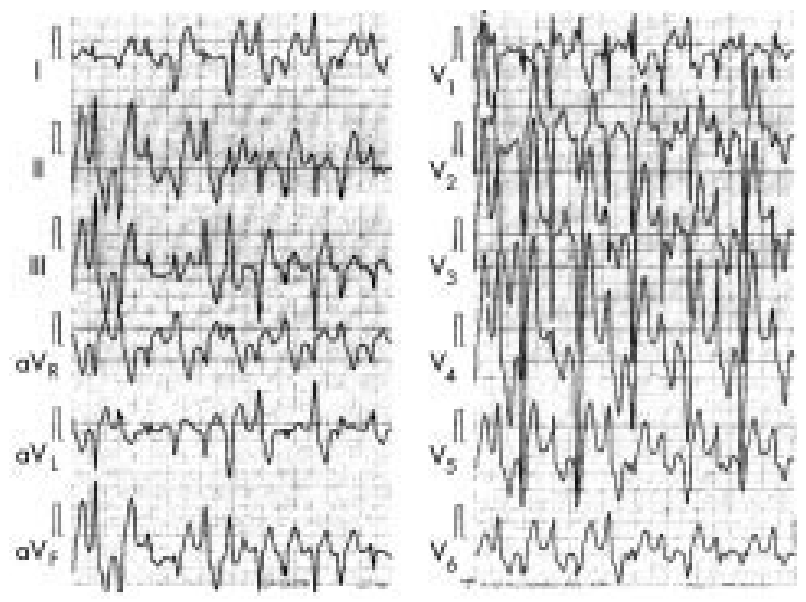

Figure 3 Bidirectional ventricular tachycardia. This is the treadmill exercise ECG of a 12 year old boy who had syncope. Note the typical bidirectional ventricular tachycardia recorded in leads V5 and aVR; however, leads aVL and V1 showed polymorphic ventricular tachycardia. See text for further discussion.

ventricular apex in seven cases, left ventricular outflow tract in five cases, right ventricular apex in three cases, and right ventricular outflow tract in eight cases (figs 1 and 2).

The mean CPVT heart rate was 192 (30) beats/min (range 150-250 beats/min), with most CPVT cases being nonsustained (20 cases, 69\%; mean ventricular tachycardia of 14 beats); six cases $(21 \%)$ were sustained, one case $(3 \%)$ was sustained with development of ventricular fibrillation, and two cases $(7 \%)$ presented with ventricular fibrillation.

The CPVT was polymorphic in 18 cases $(62 \%)$, polymorphic and bidirectional in six cases $(21 \%)$, bidirectional in three cases $(10 \%)$, and polymorphic with ventricular fibrillation in two cases (7\%). The QRS morphology characteristic of bidirectional ventricular tachycardia changed every other beat, typically with inferior and superior axis or right bundle branch block and left bundle branch block patterns. However, this bidirectional ventricular tachycardia depended strongly on the recording leads (fig 3). We also noted that the QRS morphology changed during typical bidirectional ventricular tachycardia.

CPVT was recorded by ECGs at rest in six of 23 cases (26\%) and ambulatory ECGs in 16 of 23 cases (69\%).

CPVT was induced by exercise in all 27 cases (100\%) and by catecholamine infusion in 12 of 16 cases $(75 \%)$. In the 18 cases in which it was tried, programmed stimulation entirely failed to induce CPVT. Signal averaged ECGs were recorded in 10 cases; however, a late potential was not recorded in any of these.

\section{Treatment}

Medical treatment was based on the response during the exercise test. Most patients received $\beta$ blockers (28 of 29 cases): 24 patients received propranolol, three atenolol, and one carteolol. Verapamil and a $\beta$ blocker were used in two sporadic cases; however, this combination did not completely suppress the CPVT. Mexiletine was used in three cases from an empiric standpoint. Two of these three patients took mexiletine in combination with a $\beta$ blocker. One patient had used disopyramide in combination with a $\beta$ blocker.

The acute effect of verapamil was tested in three cases of familial CPVT. Verapamil did not completely suppress CPVT; however, the endurance time was prolonged and the number of CPVT episodes decreased (fig 4).

Catheter ablation of the CPVT onset focus was used in two cases but proved unsuccessful. Only one patient did not receive drug treatment (because of severe anoxic brain damage after resuscitation from ventricular fibrillation). 

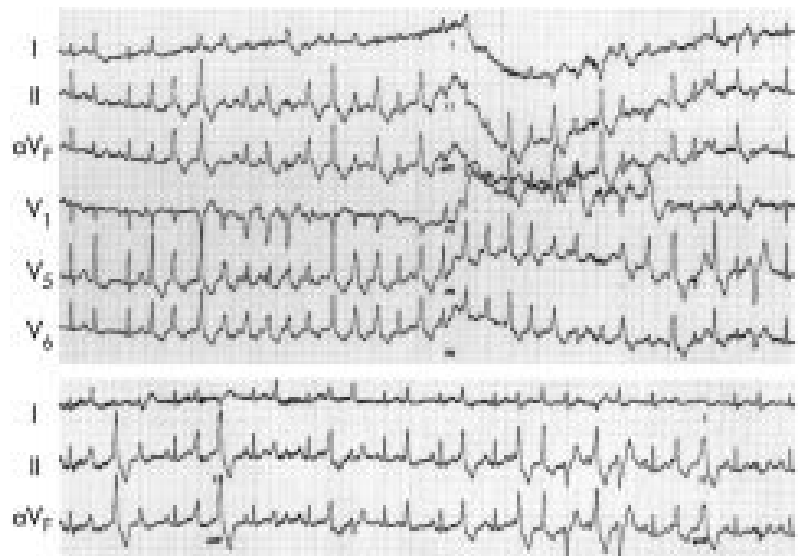

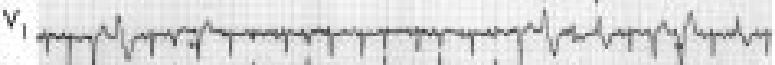

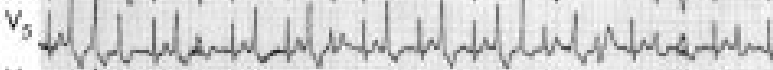

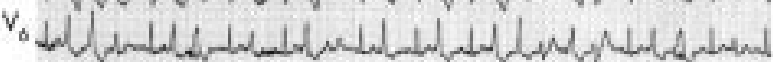

Figure 4 Effect of verapamil in familial CPVT. The ECG shows maximal treadmill exercise tests before (top) and after intravenous injection of $10 \mathrm{mg}$ of verapamil (bottom) in a 33 year old woman with familial CPVT. This patient received no drug treatment during the control state. Top: control state, 25 beats of CPVT were induced at $1 \mathrm{~min} 24 \mathrm{~s}$ after the start of the exercise. Bottom: after injection of verapamil, the endurance time was prolonged and five beats of CPVT were induced. Note the left bundle branch block and the inferior axis type premature ventricular conduction which is the trigger of this polymorphic ventricular contraction.

\section{Prognosis}

During a follow up period of 6.8 (4.9) years, seven of the patients $(24 \%)$ died, two contracted anoxic brain damage $(7 \%), 11(38 \%)$ were controlled by drug treatment, and nine $(31 \%)$ were uncontrolled by drug treatment. The 11 cases controlled by medication had no syncope or dizziness. In the nine uncontrolled cases, seven showed decreased episodes of dizziness and one a decreased ventricular tachycardia rate; one case showed no change. Two of the seven cases of sudden death received less than $1 \mathrm{mg} / \mathrm{kg}$ of propranolol, while two received more than $2 \mathrm{mg} / \mathrm{kg}$ of propranolol. The mean dose of propranolol was $52(42) \mathrm{mg}$ in the sudden death cases, 64 (37) $\mathrm{mg}$ in the controlled cases, and 56 (24) $\mathrm{mg}$ in the uncontrolled cases. These differences were not significant.

Two patients given mexiletine died during the follow up period. One of these was a 12 year old boy who was found dead at school. His treatment had recently been changed from $15 \mathrm{mg}$ of propranolol to $300 \mathrm{mg}$ of mexiletine. The other case was a 13 year old boy who had $40 \mathrm{mg}$ of propranolol and $250 \mathrm{mg}$ of mexiletine. He sometimes forgot to take his drugs and died suddenly. In another case, a $\beta$ blocker was changed to flecainide; however, because of frequent episodes of syncope, that patient was returned to $\beta$ blocker treatment.

Table 1 shows the prognosis and drug treatment in the study population, excluding the single patient who received no drugs because of severe anoxic brain damage. $\beta$ Blockers did not completely prevent sudden death. While a calcium

\begin{tabular}{lllll} 
Table 1 & \multicolumn{4}{c}{ Prognosis in relation to drug treatment } \\
\hline & $\beta$ Blocker & Ca blocker & Na blocker & Total \\
\hline Alive & 17 & 3 & 1 & 21 \\
Dead & 4 & 0 & 3 & 7 \\
Total & 21 & 3 & 4 & 28 \\
\hline
\end{tabular}

Ca blocker, calcium channel blocker; Na blocker, sodium channe blocker.

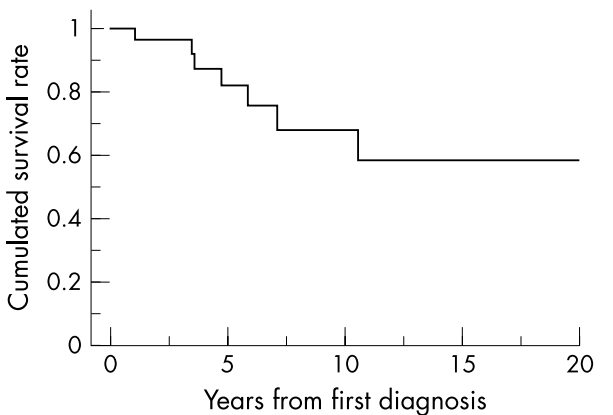

Figure 5 Cumulative survival rates. Most sudden deaths occurred within 10 years of CPVT diagnosis.

channel blocker did not worsen the prognosis, sodium channel blockers given alone or in combination with a $\beta$ blocker appeared to cause sudden death $(p=0.035)$ (two of the three cases given mexiletine and one case given disopyramide died suddenly).

The cumulative survival rate is shown in fig 5. Most cases of sudden death occurred within one decade of the first detection of CPVT. Sudden death occurred after drug treatment was discontinued by the patient in two cases, during exercise in two cases, while bathing in one case, while at school in one case (treatment had just been changed to mexiletine alone), and in one case the circumstances were unknown.

Resting ECGs and the characteristics of the ventricular tachycardia were compared between the surviving patients and those experiencing sudden death (table 2). The QRS duration of the CPVC was significantly prolonged in cases of sudden death $(\mathrm{p}=0.047)$.

\section{Inheritance}

There were two familial cases in the 25 families of the 29 patients $(8 \%)$. Based on family histories, there was a strong suggestion of an autosomal dominant inheritance.

\section{DISCUSSION}

CPVT is one of the most malignant forms of ventricular arrhythmia. On the basis of the clinical features, resting ECG patterns, and CPVT characteristics, our cases were quite similar to those of Leenhardt ${ }^{10}$; however, the prognosis was different.

From a clinical standpoint, syncope during exercise or during sympathetic activation is the key to this disease. Ninety three per cent of the CPVT patients in the present investigation experienced syncope or cardiac arrest. Exercise could induce CPVT in all patients, but unless CPVT deteriorated to ventricular fibrillation, syncope did not occur. It seems likely that most patients with CPVT experience ventricular fibrillation during syncope-in other words, without treatment patients with CPVT are prone to ventricular fibrillation and sudden death.

A normal QT interval and sinus bradycardia were characteristic resting ECG features of CPVT. There was no prolongation of the QT interval during exercise, and there was no ST-T change before CPVT onset or ventricular fibrillation. These characteristics are different from congenital long QT syndrome and the Brugada syndrome. ${ }^{15}$ In addition, the coupling interval and the induction pattern of polymorphic ventricular tachycardia was different from the short coupled variant torsade de pointes. ${ }^{16}$ Polymorphic or bidirectional ventricular tachycardia may develop in association with a prolonged QT interval. ${ }^{17-19}$ When provoked by exercise, it is unclear whether the resulting polymorphic ventricular tachycardia is the same clinical entity and has the same mechanism with a long QT interval as without.

CPVT was induced by exercise or catecholamine infusion. However, programmed stimulation could not induce CPVT. 
Table 2 Comparison of ECG measurements obtained in surviving patients and in victims of sudden death

\begin{tabular}{|c|c|c|c|c|c|}
\hline & \multicolumn{2}{|c|}{ Survivors } & \multicolumn{2}{|c|}{ Sudden death } & \multirow[b]{2}{*}{$\mathrm{p}$ Value } \\
\hline & $\mathrm{n}$ & Mean (SD) & $\mathrm{n}$ & Mean (SD) & \\
\hline QRS duration (s) & 22 & $0.071(0.012)$ & 7 & $0.073(0.014)$ & 0.765 \\
\hline QRS axis (degrees) & 22 & $45(35)$ & 7 & 57 (12) & 0.622 \\
\hline PR interval (s) & 22 & $0.135(0.023)$ & 7 & $0.131(0.017)$ & 0.873 \\
\hline QT interval (s) & 22 & $0.405(0.33)$ & 7 & $0.422(0.037)$ & 0.447 \\
\hline QTc (s) & 21 & $0.400(0.025)$ & 7 & 0.405 (0.019) & 0.542 \\
\hline HR (beats/min) & 21 & $59.4(10.4)$ & 7 & $56.4(12.0)$ & 0.353 \\
\hline PVC QRS duration (s) & 22 & $97.7(17.7)$ & 7 & 112.9 (16.0) & 0.047 \\
\hline VT rate (beats/min) & 22 & $190(29)$ & 7 & $196(34)$ & 0.701 \\
\hline
\end{tabular}

The late potential in the signal averaged ECG was negative. These findings strongly suggest that catecholamine sensitive automaticity functions as the mechanism of CPVT. Aberrant conduction from a single focus or triggered activity that may cause ventricular tachycardia, resembling torsade de pointes, cannot be ruled out. However, the change in QRS morphology is not gradual but abrupt, and in this it is very different from torsade de pointes.

With respect to the morphology of bidirectional ventricular tachycardia, we showed that the recording lead was important. If the maximal QRS vector changed in one lead during bidirectional ventricular tachycardia, the axis perpendicular to the former lead showed polymorphic ventricular tachycardia. Thus the morphology of ventricular tachycardia, which is polymorphic or bidirectional, is strongly dependent on the ECG recording lead. However, unlike other polymorphic ventricular tachycardias such as torsade de pointes, the QRS morphology is not chaotic but has some regularity. It is difficult to determine whether the morphology of bidirectional ventricular tachycardia is derived from multiple ventricular foci. An easier explanation is that the conduction direction changes with every other beat-from a single focus, one focus triggers another focus or double foci interfere with each other. However, from the programmed stimulation study, it is difficult to conclude that one focus triggers another, and the likelihood that double foci have almost the same tachycardia rate is small. The QRS morphology of bidirectional ventricular tachycardia is inconsistent in the same recording lead, so we feel that the focus of the arrhythmia cannot be pinpointed but may vary to some extent. focus may be situated in the ventricle. CPVT onset is mostly of single or double origin and usually originates from the right ventricular outflow tract, while the ensuing beat tends to originate from the left ventricle; however, Maia and colleagues reported that the majority had a left posterior inferior origin. ${ }^{20}$ If the triggering focus is from one site, successful catheter ablation is possible.

Sudden death in cases of polymorphic ventricular tachycardia is associated with a longer QRS duration than in surviving patients. It has been reported that tachycardia originating close to the proximal conduction system or the ventricular septum has a narrow QRS, ${ }^{21}$ suggesting that the onset focus in sudden death cases may lie distant from the normal conduction system. In both the sudden death group and the surviving group of our cohort, the onset focus was close to the HisPurkinje system; however, in the sudden death cases it was further from the His-Purkinje system and the interventricular septum than in the surviving cases.

A recent study suggested that familial CPVT is a genetic disorder. The chromosome involved was located in lq42-q43 in patients with an autosomal dominant inheritance ${ }^{22}$ and in 1q31-21 in patients with an autosomal recessive inheritance. More recently, ryanodine receptor gene (RyR2) mutations were found in the autosomal dominant form of familial CPVT. $^{23}{ }^{24}$ In patients with malignant hyperthermia ${ }^{25}$ and central core disease, ${ }^{27}$ an $\mathrm{MH} / \mathrm{CCD}$ gene mutation was previously found. A mutation of the RyRl receptor results in hypersensitivity to an inward calcium current. In an analogous fashion, Priori and colleagues suggested that RyR2 gene mutation might increase sensitivity to calcium ions and lead to $\mathrm{Ca}^{2+}$ release from the sarcoplasmic reticulum. ${ }^{23}$ This may increase the delayed afterdepolarisation, causing triggered activity and ventricular tachycardia. As Laitinen and colleagues suggested, ${ }^{24}$ this autosomal type of CPVT may suppress the induction of ventricular tachycardia by blocking the L type calcium channel. We showed that a calcium channel blocker suppressed some of the autosomal dominant forms of CPVT, which supports the above suggestions.

Some families with arrhythmogenic right ventricular dysplasia (ARVD) show similarities in the chromosome abnormality of $1 \mathrm{q} 42-43{ }^{28-31}$ A ryanodine receptor abnormality was also reported. The different clinical features of ARVD and CPVT are obvious. The mechanism of ARVD is re-entry, and the late potential is positive. However, as suggested in the present study, most cases of CPVT originate in the right ventricular outflow tract.

CPVT onset occurs during childhood and adolescence. None of the patients experienced syncope during infancy. There are two possible explanations for these results. The first is the intensity of exercise. In infancy, the amount of catecholamine during physical activity is not sufficient to cause CPVT because the activity is not strenuous enough. When children enter school, the possibility of syncope occurring is greater because they have many opportunities to run, play sports, and exercise. The other possibility could be the development of the ryanodine receptor. It has been reported that the ryanodine receptors are underdeveloped during infancy. ${ }^{32}$ Infants often go into cardiac arrest when they receive a calcium channel blocker. It has been suggested that immature ryanodine receptors are highly sensitive to calcium channel blockers. When infants receive a calcium channel blocker, $\mathrm{Ca}^{2+}$ cannot be released from the sarcoplasmic reticulum, so the myocardium can no longer contract. It is easy to theorise that the effects of ryanodine receptor hypersensitivity may be cancelled by receptor immaturity.

Only a few cases of CPVT have been reported in adult patients; indeed, there is a decrease in the number of episodes of syncope when the patients become adults. There are several explanations for this. For example, people tend to become more sedentary after graduation from school, with fewer opportunities for vigorous exercise. Affected individuals may also refrain from exercise to protect themselves from syncopal experiences. In addition, some children with severe forms of CPVT die during childhood, while those with benign forms of CPVT survive. Figure 5 shows how the likelihood of sudden death decreases significantly with increasing age.

$\beta$ Blockers completely controlled CPVT in only $41 \%$ of our patients, and $22 \%$ died during follow up. A sodium channel blocker was prescribed in five cases (mexiletine in three and 
disopyramide and flecainide in one case each). Three of these patients died suddenly and one had syncopal attacks. Although amiodarone was not used, these results suggest that drug treatment has only limited success.

If an electrical disorder can easily cause ventricular fibrillation, an implanted cardioverter-defibrillator (ICD) combined with $\beta$ blocker or calcium channel blocker treatment may well be the best choice for patients with CPVT. However, it remains unclear whether adult patients with CPVT need an ICD.

\section{Study limitations}

The identification of the origin of ventricular tachycardia from 12 lead ECGs is difficult. The origin was usually considered to be in the right ventricle when the QRS morphology showed a left bundle branch block pattern and in the left ventricle when a right bundle branch block pattern was observed. However, in some cases left bundle branch block patterns originate from the left ventricle, as confirmed by radiofrequency catheter ablation. In this multicentre study, endocardial mapping and catheter ablation were tried in only two patients. Therefore, we schematically identified from the bundle branch block pattern and the QRS axis four ventricular areas that were likely to form the origin of the ventricular tachycardia. Figure 1 may not represent the precise origin of the ventricular tachycardia.

With regard to the sodium channel blocker and prognosis, only four of our patients received a sodium channel blocker and three of these died suddenly. From this limited number, it is difficult to conclude that sodium channel blockers worsen the prognosis.

\section{ACKNOWLEDGEMENTS}

We thank Michio Osada (associate professor of Tsukuba University) for his assistance and comments in the preparation of the revised text.

\section{Authors' affiliations}

N Sumitomo, K Harada, Department of Paediatrics, Nihon University School of Medicine, Tokyo, Japan

M Nagashima, T Yasuda, Department of Paediatrics, Nagoya University, Japan

Y Nakamura, Department of Paediatric Cardiology, Kinki University, Japan

Y Aragaki, Department of Paediatrics, National Cardiovascular Centre, Tokyo, Japan

A Saito, K Kurosaki, Department of Cardiology, Shizuoka Children's Hospital, Japan

K Jouo, Department of Paediatrics, Kyushu Koseinenkin Hospital, Japan M Koujiro, Department of Paediatrics, University of Occupational and Environmental Health, Japan

S Konishi, Department of Paediatrics, Hiroshima University School of

Medicine, Japan

S Matsuoka, Department of Paediatrics, School of Medicine, University of Tokushima, Japan

T Oono, Department of Paediatrics, Kyushu University, Japan

S Hayakawa, Department of Paediatrics, Seirei Mikatahara General

Hospital, Japan

M Miura, Department of Cardiology, Metropolitan Kiyose Children's

Hospital, Japan

H Ushinohama, Department of Cardiology, Fukuoka Children's

Hospital, Japan

T Shibata, I Niimura, Department of Paediatrics, Yokohama City

University, School of Medicine, Japan

\section{REFERENCES}

1 Berg KJ. Multifocal ventricular extrasystoles with Adams-Stokes syndrome in siblings. Am Heart J 1960;60:966-70.

2 Horan M, Venables AW. Paroxysmal tachycardia with episodic unconsciousness. Arch Dis Child 1962;37:82-5.

3 Wennewold A, Melchior JC, Sandoe E. Adams-Stokes syndrome in children without organic heat disease: electrocardiogram after exercise as a diagnostic tool. Acta Med Scand 1965;177:557-63.

4 Dik T, Janukowicz C. Bidirectional tachycardia in a case of recurrent paroxysmal tachycardia with ventricular fibrillation. Cardiologia $1968 \cdot 52 \cdot 132-7$
5 Reid DS, Tynan M, Braidwood L, et al. Bidirectional tachycardia in a child: a study using His bundle electrography. Br Heart J 1975;37:339-44.

6 Wennevold A, Sandoe E. Paroxysmal ventricular fibrillation in children: long term follow-up of three cases treated with beta-blocking agents. Acta Med Scand 1977;202:425-7.

7 Coumel P, Fidelle J, Lucet V, et al. Catecholaminergic-induced severe ventricular arrhythmias with Adam-Stokes syndrome in children: report of four cases. Br Heart J 1978;40(suppl):28-37.

8 Shaw TR. Recurrent ventricular fibrillation associated with normal QT interval. Q J Med 1981;200:451-62.

9 Cohen TJ, Liem LB, Hancock EW. Association of bidirectional ventricular tachycardia with familial sudden death syndrome. Am J Cardiol 1989;64:1078-9

10 Leenhardt A, Lucet V, Denjoy I, et al. Catecholaminergic polymorphic ventricular tachycardia in children. Circulation 1995: 91:1512-19.

11 Eisenberg S, Scheinman M, Dullet N, et al. Sudden cardiac death and polymorphous ventricular tachycardia in patients with normal QT intervals and normal systolic cardiac function. Am J Cardiol 1995; 75:687-92.

12 Myrianthefs $M$, Cariolou $M$, Eldar $M$, et al. Exercise-induced ventricular arrhythmias and sudden cardiac death in a family. Chest 1997;111:1130-3.

13 Brooks R, Burgess J. Idiopathic ventricular tachycardia. A review. Medicine 1988;67:271-94

14 Belhassen B, Viskin S. Idiopathic ventricular tachycardia and fibrillation. J Cardiovasc Electrophysiol 1993:4:356-68.

15 Brugada $\mathbf{P}$, Brugada J. Right bundle branch block, persistent ST segment elevation and sudden cardiac death: a distinct clinical and electrocardiographic syndrome. A multicenter report. J Am Coll Cardiol 1992;20:1391-6.

16 Leenhardt A, Glaser E, Burguera M, et al. Short-coupled variant of torsade de pointes. A new electrocardiographic entity in the spectrum of idiopathic ventricular tachycardia. Circulation 1994; 89:206-15.

17 von Bernuth G, Bernsau U, Gutheil H, et al. Tachyarrhymic syncope in children with structurally normal hearts with and without QT-prolongation in the electrocardiogram. Eur J Pediatr 1982;138:206-10.

18 Glikson M, Constantini N, Graftstein Y, et al. Familial bidirectional ventricular tachycardia. Eur Heart J 1991:12:741-5.

19 Noh Cl, Song JY, Kim HS, et al. Ventricular tachycardia and exercise related syncope in children with structurally normal hearts: emphasis on repolarisation abnormality. Br Heart J 1995;73:544-7.

20 Maia IG Cruz Filho F, La Fragundes $M$, et al. Identification of origin of the ventricular beats present within the bursts of bi-directional tachycardia and in the short coupling variant of torsade de pointes by 12-lead ECG and electrophysiology. Eur Heart J 2001;22(suppl):364.

21 Hayes JJ, Stewart RB, Green HL, et al. Narrow QRS ventricular tachycardia. Ann Intern Med 1991;114:460-3.

22 Swan H, Piippo K, Viitasalo M, et al. Arrhythmic disorder mapped to chromosome 1q42-q43 causes malignant polymorphic ventricular tachycardia in structurally normal hearts. J Am Coll Cardiol 1999:34:2035-42.

23 Priori SG. Napolitano C, Tiso N, et al. Mutations in the cardiac ryanodine receptor gene (hRyR2) underlie catecholaminergic polymorphic ventricular tachycardia. Circulation $2001 ; 103: 196-200$.

24 Laitinen PJ, Brown KM, Piippo K, et al. Mutations of the cardiac ryanodine receptor (RyR2) gene in familial polymorphic ventricular tachycardia. Circulation 2001;103:485-90.

25 McCarthy TV, Quane KA, Lynch PJ. Ryanodine receptor mutations in malignant hyperthermia and central core disease. Hum Mutat 2000;15:410-17

26 Brown RL, Pollock AN, Couchman KG, et al. A novel ryanodine receptor mutation and genotype-phenotype correlation in a large malignant hyperthermia New Zealand Maori Pedigree. Hum Mol Genet 2000;9:1514-24.

27 Lynch PJ, Tong J, Lehane $M$, et al. A mutation in the transmembrane luminal domain of the ryanodine receptor is associated with abnormal calcium release channel function and severe central core disease. Proc Natl Acad Sci USA 1999;96:4164-9.

28 Rampazzo A, Nava A, Danieli GA, et al. The gene for arrhythmogenic right ventricular cardiomyopathy maps to chromosome 14q23-q24. Hum Mol Genet 1994;3:959-62.

29 Rampazzo A, Nava A, Erne P, et al. A new locus for arrhythmogenic right ventricular cardiomyopathy (ARVD2) maps to chromosome lq42-q43. Hum Mol Genet 1995;4:2151-4.

30 Rampazzo A, Nava A, Miorin M, et al. ARVD4, a new locus for arrhythmogenic right ventricular cardiomyopathy, maps to chromosome 2 long arm. Genomics 1997;45:259-63.

31 Severini GM, Krajinovic M, Pinamonti B, et al. A new locus for arrhythmogenic right ventricular dysplasia on the long arm of chromosome 14. Genomics 1996;31:193-200.

32 Sedarat F, Xu L, Moore EDW, et al. Localization of dihydropyridine and ryanodine receptors in neonate rabbit heart using confocal microscopy. Am J Physiol Heart Circ Physiol 2000;279:H202-9. 